\title{
Improving the Computational Efficiency of the Successive Projections Algorithm by using a Sequential Regression Implementation: A Case Study Involving NIR Spectrometric Analysis of Wheat Samples
}

\author{
Anderson S. Soares, ${ }^{a}$ Arlindo R. Galvão Filho, ${ }^{a}$ Roberto K. H. Galvão ${ }^{a}$ and \\ Mário César U. Araújo*,b \\ aInstituto Tecnológico de Aeronáutica, Divisão de Engenharia Eletrônica, \\ 12228-900 São José dos Campos-SP, Brazil \\ ${ }^{b}$ Universidade Federal da Paraíba, CCEN, Departamento de Química, CP 5093, \\ 58051-970 João Pessoa-PB, Brazil
}

\begin{abstract}
Este artigo propõe uma implementação de regressões seqüenciais para o algoritmo das projeções sucessivas (APS), que é uma técnica de seleção de variáveis para regressão linear múltipla. Para ilustração, apresenta-se um exemplo envolvendo a determinação de proteína em trigo por espectrometria no infravermelho próximo. As previsões do modelo resultante exibiram um coeficiente de correlação de 0.989 e um RMSEP (erro médio quadrático de predição) de $0.2 \% \mathrm{~m} / \mathrm{m}$ na faixa de $10.2-16.2 \% \mathrm{~m} / \mathrm{m}$. A implementação proposta proporcionou ganhos computacionais de até cinco vezes.
\end{abstract}

This short report proposes a sequential regression implementation for the successive projections algorithm (SPA), which is a variable selection technique for multiple linear regression. An example involving the near-infrared determination of protein in wheat is presented for illustration. The resulting model predictions exhibited a correlation coefficient of 0.989 and an RMSEP (rootmean-square error of prediction) value of $0.2 \% \mathrm{~m} / \mathrm{m}$ in the range $10.2-16.2 \% \mathrm{~m} / \mathrm{m}$. The proposed implementation provided computational gains of up to five-fold.

Keywords: successive projections algorithm, multivariate calibration, sequential regressions, computational efficiency, near-infrared spectrometry, wheat

\section{Introduction}

The successive projections algorithm (SPA) is a variable selection technique designed to minimize multicollinearity problems in multiple linear regression (MLR). ${ }^{1}$ In several applications concerning UV-Vis,,${ }^{1,2}$ ICP-OES, ${ }^{3}$ FT-IR ${ }^{4}$ and NIR spectrometry, ${ }^{4-8}$ SPA was found to provide models with good predictive performance. It has also been successfully employed in other fields such as QSAR (quantitative structure activity relationships $)^{9}$ and classification. ${ }^{10,11}$ A graphic user interface for SPA is freely available at $<$ http://www.ele.ita.br/ kawakami/spa>.

SPA comprises three main phases. ${ }^{7,12}$ Phase 1 consists of projection operations carried out on the matrix of instrumental responses. These projections are used to generate chains of variables with successively more

\footnotetext{
*e-mail: laqa@ quimica.ufpb.br
}

elements. Each element in a chain is selected in order to display the least collinearity with the previous ones. In Phase 2, candidate subsets of variables are extracted from the chains and evaluated according to the predictive performance of the resulting MLR model. Such a performance can be assessed by using cross-validation or a separate validation set. ${ }^{13}$ Finally, Phase 3 consists of a variable elimination procedure aimed at improving the parsimony of the model. ${ }^{7,12}$

Due to the need of building an MLR model for each subset of variables under consideration, Phase 2 may be considerably more demanding, in computational terms, as compared to Phases 1 and 3. For example, in a problem involving 389 calibration samples, 193 validation samples and 690 variables, Phases 1, 2 and 3 account for 1.9, 98.1 and $0.02 \%$ of the total time, respectively. These results were obtained by using the setup described in the Experimental section and may be slightly different if 
another configuration is employed. However, it is clear that Phase 2 is the holdup for the overall computational efficiency of SPA.

The present work is aimed at improving the efficiency of Phase 2 in SPA by using a sequential regression procedure proposed elsewhere. ${ }^{14}$ The computational gain thus obtained is demonstrated in an example involving a large NIR dataset of wheat samples.

\section{Background and theory}

\section{Notation}

Matrices are represented by bold capital letters and scalars by italic characters. The matrix $\mathbf{X}$ of instrumental response data has dimensions $(N \times K)$, where $N$ and $K$ denote the number of calibration samples and spectral variables, respectively. The $k^{\text {th }}$ column of $\mathbf{X}$ corresponds to variable $x_{k}$. Phase 2 of SPA evaluates subsets with one up to $M$ variables, where $M$ can be specified by the analyst under the restriction $M \leq \min (N-1, K)$ for mean-centered data. The hat symbol $\left({ }^{\wedge}\right)$ indicates an estimated value.

\section{Successive projections algorithm}

Phase 1 of SPA consists of projection operations involving the columns of matrix $\mathbf{X}$. These operations are used to form $K$ chains with $M$ variables each. The first element of the $k^{\text {th }}$ chain corresponds to $x_{k}$. Each subsequent element in the chain is selected in order to display the least collinearity with the previous ones. ${ }^{1}$

In Phase 2, each chain is used to define $M$ candidate subsets of variables. The $m^{\text {th }}$ subset corresponds to the $m$ first variables in the chain. These candidate subsets are then evaluated according to a suitable criterion that takes into account their relation with the dependent variable $y$, which was not employed in the projection operations. For this purpose, metrics associated to the prediction ability of the resulting MLR model can be employed. ${ }^{7}$

The third and final phase is a backward elimination procedure aimed at discarding variables that do not significantly contribute towards the prediction ability of the MLR model. ${ }^{7,12}$

\section{Sequential regressions formulation}

Without loss of generality, let us assume that $\left\{x_{1}, x_{2}\right.$, $\left.\ldots, x_{M}\right\}$ (in this order) is a chain of variables obtained in Phase 1 of SPA. In Phase 2, these variables are used to obtain $M$ progressively larger MLR models, starting from a single-variable $\left(x_{1}\right)$ model, followed by models with two $\left(x_{1}, x_{2}\right)$, up to $M\left(x_{1}, x_{2}, \ldots, x_{M}\right)$ variables. Each of these models can be obtained by a least-squares regression procedure, independently from the others. Such a process requires the inversion of progressively larger matrices. ${ }^{15}$ The sequential regressions formulation described below reduces computational workload by avoiding the need for such inverse calculations.

The sequential regressions formulation starts from a single-variable model of the form $y=\beta_{1}^{(1)} x_{1}+\varepsilon^{y x_{1}}$, where $\beta_{1}^{(1)}$ is the regression coefficient and $\varepsilon^{y x_{1}}$ is the residue. An offset term is not included, under the assumption that the data have been mean-centered. Superscripts (1) and $y \mid x_{1}$ denote that one independent variable is employed in the model and that $y$ is regressed on $x_{1}$, respectively. The least-squares estimate of $\beta_{1}^{(1)}$ is given by ${ }^{15}$

$\hat{\beta}_{1}^{(1)}=\frac{\sum_{i=1}^{N} y_{i} x_{i, 1}}{\sum_{i=1}^{N}\left(x_{i, 1}\right)^{2}}$

where $y_{i}, x_{i, 1}$ represent the values of $y$ and $x_{1}$ for the $i^{\text {th }}$ calibration object, respectively.

By using a similar notation, the two-variable model is written as $y=\beta_{1}^{(2)} x_{1}+\beta_{2}^{(2)} x_{2}+\varepsilon^{y x_{1}, x_{2}}$. In order to obtain $\hat{\beta}_{1}^{(2)}$ and $\hat{\beta}_{2}^{(2)}, x_{2}$ is initially regressed on $x_{1}$ according to a model of the form

$x_{2}=\delta_{1}^{x_{1} \mid x_{1}} x_{1}+\varepsilon^{x_{2} \mid x_{1}}$

The coefficient estimate $\hat{\delta}_{1}^{x_{2} \mid x_{1}}$ can be calculated by univariate regression as

$$
\hat{\delta}_{1}^{x_{2} x_{1}}=\frac{\sum_{i=1}^{N} x_{i, 2} x_{i, 1}}{\sum_{i=1}^{N}\left(x_{i, 1}\right)^{2}}
$$

Then, as shown elsewhere, ${ }^{14} \hat{\beta}_{1}^{(2)}$ and $\widehat{\beta}_{2}^{(2)}$ can be obtained as

$\hat{\beta}_{2}^{(2)}=\frac{\sum_{i=1}^{N} e_{i}^{y \mid x_{1}} x_{i, 2}}{\sum_{i=1}^{N} e_{i}^{x_{2} \mid x_{1}} x_{i, 2}}, \hat{\beta}_{1}^{(2)}=\hat{\beta}_{1}^{(1)}-\hat{\delta}_{1}^{x_{2} \mid x_{1}} \hat{\beta}_{2}^{(2)}$

where

$e_{i}^{y \mid x_{1}}=y_{i}-\hat{\beta}_{1}^{(1)} x_{i, 1}$

and

$e_{i}^{x_{2} \mid x_{1}}=x_{i, 2}-\hat{\delta}_{1}^{x_{2} \mid x_{1}} x_{i, 1}$

This procedure can be generalized to obtain a model with $m$ variables from a model with $(m-1)$ variables, 
where $m$ ranges from 2 to $M$. For this purpose, the new independent variable $x_{m}$ is initially regressed on $x_{1}, x_{2}, \ldots, x_{m-1}$ according to a model of the form

$x_{m}=\delta_{1}^{x_{m} \mid x_{1}, \ldots, x_{m-1}} x_{1}+\delta_{2}^{x_{m} \mid x_{1}, \ldots, x_{m-1}} x_{2}+\cdots+\delta_{m-1}^{x_{m} \mid x_{1}, \ldots, x_{m-1}} x_{m-1}+\varepsilon^{x_{m} \mid x_{1}, \ldots, x_{m-1}}$

The $\beta$ coefficients of the $m$-variable model are then calculated as

$\hat{\beta}_{m}^{(m)}=\frac{\sum_{i=1}^{N} e_{i}^{y \mid x_{1}, \ldots, x_{k-1}} x_{i, m}}{\sum_{i=1}^{N} e_{i}^{x_{m} \mid x_{1}, \ldots, x_{m-1}} x_{i, m}}$

$\hat{\beta}_{m-j}^{(m)}=\hat{\beta}_{m-j}^{(m-1)}-\hat{\delta}_{m-j}^{x_{m} \mid x_{1}, \ldots, x_{m-1}} \hat{\beta}_{m}^{(m)}, j=1, \ldots, m-1$

where

$e_{i}^{x_{m} \mid x_{1}, \ldots, x_{m-1}}=x_{i, m}-\left(\hat{\delta}_{1}^{x_{m} \mid x_{1}, \ldots, x_{m-1}} x_{i, 1}+\hat{\delta}_{2}^{x_{m} \mid x_{1}, \ldots, x_{m-1}} x_{i, 2}+\cdots+\hat{\delta}_{m-1}^{x_{m} \mid x_{1}, \ldots, x_{m-1}} x_{i, m-1}\right)$

$e_{i}^{y \mid x_{1}, \ldots, x_{m-1}}=y_{i}-\left(\hat{\beta}_{1}^{(m-1)} x_{i, 1}+\hat{\beta}_{2}^{(m-1)} x_{i, 2}+\cdots+\hat{\beta}_{m-1}^{(m-1)} x_{i, m-1}\right)$

A similar sequential procedure ${ }^{14}$ can be employed to calculate the $\delta$ coefficients involved in the regression of $x_{m}$ on $x_{1}, x_{2}, \ldots, x_{m-1}$.

\section{Experimental}

\section{NIR data set}

The dataset employed in this work consists of 775 Vis-NIR spectra of whole-kernel wheat, which were used as shoot-out data in the 2008 International Diffuse Reflectance Conference (http://www.idrc-chambersburg. org/shootout.html). Protein content was chosen as the parameter of interest. The spectra were acquired in the range 400-2,500 $\mathrm{nm}$ with a resolution of $2 \mathrm{~nm}$. In the present work, only the NIR region in the range 1,100 $2,500 \mathrm{~nm}$ was employed. In order to remove undesirable baseline features, first derivative spectra were calculated by using a Savitzky-Golay filter with a 2 nd order polynomial and an 11-point window. ${ }^{17}$ The resulting derivative spectra comprised 690 spectral variables.

The Kennard-Stone (KS) algorithm ${ }^{18,19}$ was applied to the derivative spectra to separate data into calibration, validation and prediction sets with 389, 193 and 193 samples, respectively. The validation set was employed to guide the selection of variables in Phase 2 of SPA. The prediction set was only used in the final performance assessment of the MLR model.

\section{Computational setup}

All calculations were carried out by using a desktop computer with an Intel Core Duo processor $(2.13 \mathrm{GHz})$ and 3 GB RAM memory. The Matlab 6.5 software platform was employed throughout. The standard (i.e., non-sequential) regression calculations were done by using the left division (or "backslash") operator, which is a built-in function of Matlab. ${ }^{16}$

\section{Results and Discussion}

Figures $1 \mathrm{a}$ and $1 \mathrm{~b}$ present the original and derivative spectra, respectively. As can be seen, the original spectra presented undesirable baseline features, which were removed by the derivative procedure.
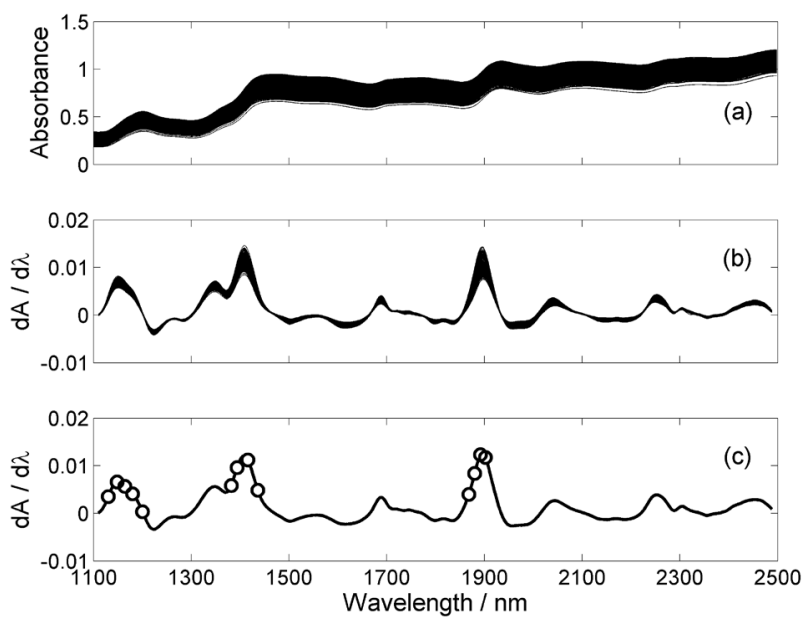

Figure 1. (a) Original and (b) derivative NIR spectra of the wheat samples. (c) Derivative spectrum of one of the samples with indication of the wavelenghts selected by SPA (circle markers).

Figure 2 presents the time required for completion of Phase 2 as a function of the maximum number $M$ of variables to be selected. For $M=200$, for instance, regressions involving one up to 200 variables were carried out. As can be seen, the computational time increases with $M$, but the increase is less pronounced if the sequential regression procedure is employed. For $M=200$, for example, the proposed procedure reduces the time by a factor of approximately three. Such a computational gain becomes even more substantial for larger $M$, rising to five for $M=388$.

By applying SPA with $M=388,13$ variables were selected, as indicated in Figure 1c. Figure 3 compares the model predictions with the reference values of protein content for the prediction set. There is good agreement between predicted and reference values, as indicated by a correlation coefficient of 0.989 . Moreover, the root-meansquare error of prediction (RMSEP) $)^{12,17}$ is $0.2 \% \mathrm{~m} / \mathrm{m}$, which 
is small as compared to the range of protein content in the prediction set $(10.2-16.2 \%, \mathrm{~m} / \mathrm{m})$.

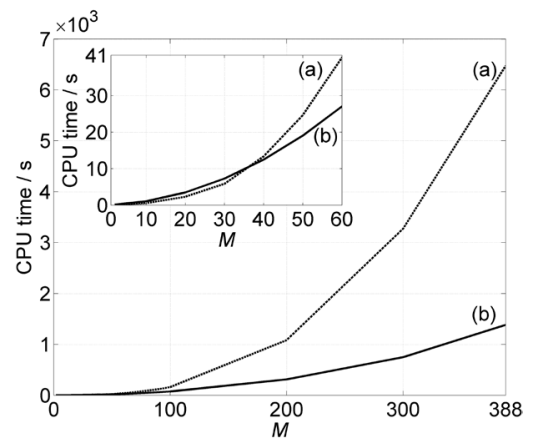

Figure 2. Time for completion of Phase 2 in SPA with (a) original and (b) proposed formulation. The inset presents an expanded view of the initial part of the graph.

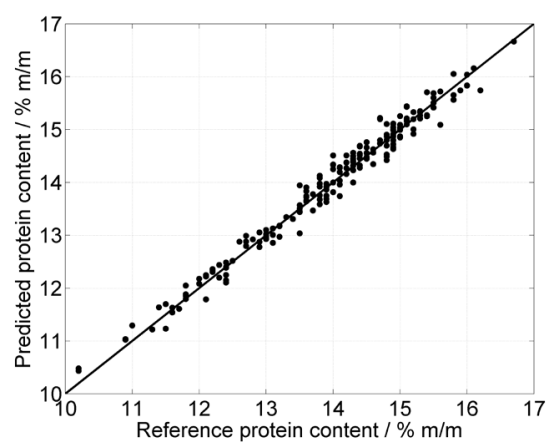

Figure 3. Predicted and reference values of protein content in the prediction set.

\section{Conclusions}

This paper proposed a new implementation of the successive projections algorithm (SPA) based on the use of a sequential regression procedure. This procedure was employed in Phase 2 of SPA, which is the computational bottleneck of the overall algorithm. For illustration, a large dataset of NIR spectra was employed for determination of protein in wheat. In this case, SPA selected 13 out of 690 spectral variables. The resulting model provided good predictions, with a correlation coefficient of 0.989 and an RMSEP value of $0.2 \% \mathrm{~m} / \mathrm{m}$ in the range $10.2-16.2 \%, \mathrm{~m} / \mathrm{m}$. The proposed sequential regression implementation provided substantial gains (up to five-fold) in computational efficiency as compared to the original SPA formulation.

\section{Acknowledgments}

This work was supported by FAPESP (Grants 2006/588506 and 2007/57803-7), CAPES (PROCAD Grant 0081/05-1) and CNPq (MSc scholarship and research fellowships).

\section{References}

1. Araújo, M. C. U.; Saldanha, T. C. B.; Galvão, R. K. H.; Yoneyama, T.; Chame, H. C.; Visani, V.; Chemom. Intell. Lab. Syst. 2001, 57, 65.

2. Dantas Filho, H. A.; Souza, E. S. O. N.; Visani, V.; Barros, S. R. R. C.; Saldanha, T. C. B.; Araújo, M. C. U. ; Galvão, R. K. H.; J. Braz. Chem. Soc. 2005, 16, 58.

3. Galvão, R. K. H.; Pimentel, M. F.; Araujo, M. C. U.; Yoneyama, T.; Visani, V.; Anal. Chim. Acta 2001, 443, 107.

4. Honorato, F. A.; Galvão, R. K. H.; Pimentel, M. F.; Neto, B. B.; Araújo, M. C. U.; Carvalho, F. R.; Chemom. Intell. Lab. Syst. 2005, 76,65 .

5. Breitkreitz, M. C.; Raimundo Jr, I. M.; Rohwedder, J. J. R.; Pasquini, C.; Dantas Filho, H. A.; José, G. E.; Araújo, M. C. U.; Analyst 2008, 128, 1204.

6. Dantas Filho, H. A. D.; Galvão, R. K. H.; Araújo, M. C. U.; Silva, E. C.; Saldanha, T. C. B.; José, G. E.; Pasquini, C.; Raimundo Jr., I. M.; Rohwedder, J. J. R.; Chemom. Intell. Lab. Syst. 2004, 72, 83.

7. Galvão, R. K. H.; Araújo, M. C. U.; Fragoso, W. D.; Silva, E. C.; José, G. E.; Soares, S. F. C.; Paiva, H. M.; Chemom. Intell. Lab. Syst. 2008, 92, 83.

8. Pereira, A. F. C.; Pontes, M. J. C.; Gambarra, N. F. F.; Santos, S. R. B.; Galvão, R. K. H.; Araújo, M. C. U.; Food Res. Int. 2008, 41,341 .

9. Akhlaghi, Y.; Kompany-Zareh, M.; J. Chemom. 2006, $20,1$.

10. Pontes, M. J. C.; Galvão, R. K. H.; Araújo, M. C. U.; Moreira, P. N. T.; Pessoa Neto, O. D.; José, G. E.; Saldanha, T. C. B.; Chemom. Intell. Lab. Syst. 2005, 78, 11.

11. Gambarra Neto, F. F.; Marino, G.; Araújo, M. C. U.; Galvão, R. K. H.; Pontes, M. J. C.; Medeiros, E. P.; Lima, R. S.; Talanta 2009, 77, 1660.

12. Galvão, R. K. H.; Araújo, M. C. U. In Comprehensive Chemomics: Chemical and Biochemical Data Analysis; Brown, S.; Tauler, R.; Walczak, B., eds.; Elsevier: Oxford, 2009.

13. Galvão, R. K. H.; Araújo, M. C. U.; Silva, E. C.; José, G. E.; Soares, S. F. C.; Paiva, H. M.; J. Braz. Chem. Soc. 2007, 18, 1580.

14. Gusnanto, A.; Pawitan, Y.; Huang, J.; J. Chemom. 2003, 17, 174.

15. Draper, N. R.; Smith, H.; Applied Regression Analysis, $3^{\text {rd }}$ ed., Wiley: New York, 1998.

16. Matlab Function Reference, vol.1, The Mathworks: Natick, MA, 2002.

17. Beebe, K. R.; Pell, R. J.; Seasholtz, B.; Chemomics - A Pratical Guide, Wiley: New York, 1998.

18. Kennard, R. W.; Stone L. A.; Technometrics 1969, 11, 137.

19. Kanduc, K. R.; Zupan, J.; Majcen N.; Chemom. Intell. Lab. Syst. 2003, 65, 221.

Received: August 19, 2009

Web Release Date: December 15, 2009

FAPESP helped in meeting the publication costs of this article. 